\title{
Mini-Mental Status Exam
}

National Cancer Institute

\section{Source}

National Cancer Institute. Mini-Mental Status Exam. NCI Thesaurus. Code C74982.

A standardized and structured interview originally developed by Marshal Folstein, Susan

Folstein and Paul McHugh (Journal of Psychiatric Research, 1975), which is used to

evaluate an individual's cog nitive function. 